\title{
RESÍDUOS DE CERÂMICA VERMELHA COMO UM MATERIAL AMBIENTALMENTE SUSTENTÁVEL PARA USO NA PAVIMENTAÇÃO
}

\section{WASTE OF RED CERAMIC AS AN ENVIRONMENTALLY SUSTAINABLE MATERIAL FOR PAVING PURPOSE}

Wana Maria de Souza

Graduanda em Engenharia Ambiental - Instituto Federal do Ceará (IFCE) - Campus Juazeiro do Norte. (wanamaria19@gmail.com)

Antonio Júnior Alves Ribeiro

Doutor em Engenharia de Transportes, Professor - Instituto Federal do Ceará (IFCE) - Campus Juazeiro do Norte. (ajar.junior@gmail.com)

Isaac Wanderson de Pontes Xavier

Graduando em Engenharia Ambiental - Instituto Federal do Ceará (IFCE) - Campus Juazeiro do Norte. (isaacxavier.wanderson@gmail.com)

Marianny Viana dos Santos

Graduanda em Construção de Edifícios - Instituto Federal do Ceará (IFCE) - Campus Juazeiro do Norte. (mariannyviana@gmail.com)

Submissão: 26 de Julho de 2019 Aceite: 24 de Setembro de 2019

\section{Resumo}

A produção e a disposição inadequada dos Resíduos da Construção Civil (RCC) têm gerado diversos impactos no meio ambiente. Uma alternativa de destinação para estes materiais é a sua aplicação nos projetos e construção de rodovias, uma vez que o uso desses resíduos contribui para a construção sustentável de estradas. Tal alternativa diminui a extração de materiais naturais em jazidas e empréstimos de materiais, bem como evita o acúmulo e disposição dos resíduos de forma ambientalmente inadequada. Um dos resíduos mais gerados na construção civil é proveniente da indústria de cerâmica vermelha (RCV). Desta forma, o escopo desta pesquisa é avaliar as propriedades físicas dos RCV puro e misturado com um solo arenoso (A-2-4), para aplicação em camadas granulares de pavimentos rodoviários, através de ensaios tradicionalmente empregados na pavimentação. Para tanto, foram analisados o solo puro, RCV puro e solo com RCV, utilizando taxas de substituição de 30\%, 50\% e 70\%. Os resultados revelaram que os RCV têm potencial de emprego em camadas de base, sub-base e reforço do subleito em pavimentos rodoviários, considerando as especificações rodoviárias brasileiras vigentes.

Palavras-chave: Pavimentos; Materiais Alternativos; Materiais sustentáveis; Solos.

\section{Abstract}

The inadequate production and disposal of construction waste (CW) has an impact on the environment. Projects and road construction are alternative destinations for these materials, as their use can contribute to the sustainable construction of roads. The use of these waste can help reduce the extraction of raw materials in deposits, also preventing the accumulation and disposal of waste in an environmentally inappropriate. One of the most generated waste in the construction industry comes from the red ceramic industry, they can be called red ceramic waste $(\mathrm{RCW})$. Thus, the aims of this research is to evaluate the properties of RCW and RCW mixed with soil of the A-2-4 class, for application how road material and pavements design, through tests generally used in paving. Were analyzed clean soil, clean RCW and soil mixed with $\mathrm{RCW}$, using replacement rates of $30 \%, 50 \%$ and $70 \%$. Further, of the results of the test show that the RCW have the potential to be use how base, subbase and subgrade reinforcement of the pavement. Consequently, this research suggests to use RCW as an alternative material to improve the performance of soil in an economically and environmentally beneficial way.

Keywords: Pavements; Alternative road materials; Sustainable road materials; Soils. 


\section{INTRODUÇÃO E FUNDAMENTAÇÃO TEÓRICA}

A indústria de cerâmica brasileira é responsável por contribuir com, aproximadamente, $1 \%$ no PIB do país, sendo $40 \%$ desta contribuição representada pelo setor de cerâmica vermelha. Esse setor gera cerca de 130 milhões de toneladas de cerâmica vermelha e 6,5 milhões de toneladas de resíduos anualmente (GARCIA, 2014). Em geral, os resíduos dessas indústrias são oriundos de peças que não atenderam as características geométricas adequadas para comercialização ou que sofreram quebras durante a produção (REDIVO, 2011).

Para Souza et al. (2018), o índice de perdas nas indústrias de cerâmica vermelha varia entre $5 \%$ e $20 \%$, a depender do tipo de indústria, considerando das mais modernas às mais antigas. No Brasil, estima-se que o valor médio de perdas seja de 10\%, em decorrência da diversidade das cerâmicas. Já no estado do Ceará, esse percentual sobe para $20 \%$, um valor equivalente a, aproximadamente, $400 \mathrm{~kg}$ de Resíduo de Cerâmica Vermelha (RCV) por milheiro de peças fabricadas.

De acordo com o Banco do Nordeste do Brasil (2010), o estado do Ceará produz 352.000 milheiros de peças por mês, e a região do Cariri cearense, em torno de 70.506 milheiros por mês ( $20 \%$ da produção do estado). Considerando $20 \%$ de perda, Ceará e Cariri geram mensalmente 141.012 e 28.202 toneladas de RCV, respectivamente.

Cabalar, Hanssan e Abdulnafaa (2016) citaram que as propriedades dos RCV e de outros resíduos de construção civil (RCC) têm sido exploradas em diversas aplicações na engenharia, sendo este setor uma boa alternativa de reciclagem para tais materiais.

Algumas pesquisas têm mostrado que a substituição total ou parcial de agregados pétreos por resíduos tem melhorado a qualidade de concretos e argamassas (ALVES et al., 2014; PACHECO-TORGAL; JALALI, 2010; BINICI, 2007; DE BRITO; PEREIRA, 2005). Já outros pesquisadores têm buscado utilizar os resíduos mais finos (fíler) como materiais pozolânicos na produção de Cimento Portland ou como adições em concretos e argamassas (GARCIA et al., 2015; GARCIA, 2014; SALES; ALFERES FILHO, 2014; PEREIRA et al., 2015; OLIVEIRA; GOMES;
SANTOS, 2012; BETAT; PEREIRA; VERNEY, 2009).

Silva et al. (2017) analisaram o uso de resíduos de cerâmica vermelha para o melhoramento de solos para aplicação em Rodovias de Baixo Volume de Tráfego (RBVT) no estado de Tocantins. Seus resultados mostraram que a adição dos RCV melhorou as características mecânicas do solo estudado. Ossa, Garcí e Botero (2016) testaram a substituição de agregados pétreos por RCC em misturas asfálticas para pavimentos urbanos. Eles concluíram que as deformações permanentes das misturas asfálticas com até $20 \%$ de substituição não aumentaram em comparação com as misturas convencionais, podendo, assim, os RCC serem aplicados nessa proporção. Já Herrador (2012) construiu trechos experimentais de pavimentos com RCC como camadas de base e fez medidas de deflexões por meio do Falling Weight Deflectomete (FWD). Ele constatou que os pavimentos com RCC tiveram melhor desempenho frente aos pavimentos com materiais pétreos, usando como comparativo as bacias de deflexões geradas por meio de FWD. Leite et al. (2011) obtiveram bons resultados de capacidade de suporte, módulo de resiliência e deformação permanente de RCC como agregados graúdos em camadas de base e subbase de pavimentos de RBVT.

Pode-se verificar que a utilização de resíduos oriundos da construção civil em camadas de pavimentos mostra-se como uma alternativa técnica viável. Este trabalho tem por objetivo identificar o potencial uso dos resíduos de cerâmica vermelha (RCV), provenientes da Região Metropolitana do Cariri (RMC), em camadas estruturais dos pavimentos rodoviários. Com isso, pretende-se contribuir para construção sustentável de rodovias, por meio do reúso de resíduos, atenuando a dependência e os impactos causados pela extração de materiais naturais e pela disposição ambientalmente inadequada dos RCC.

\section{METODOLOGIA}

Os materiais utilizados na pesquisa foram RCV e um solo do tipo areno-siltoso. O RCV foi obtido nas áreas de descarte de resíduos de quatro (04) cerâmicas na RMC. Os resíduos coletados estavam no formato de tijolos cerâmicos (paralelepípedo) fraturados ou 
danificados. Já o solo usado nos experimentos de laboratório foi extraído de uma jazida no município de Juazeiro do Norte, também localizado na RMC.

Com o intuito de se obter material granular a partir do RCV, o resíduo coletado foi triturado em um compactador mecânico, de tal maneira que o resíduo passasse na peneira de abertura 19,00mm. Em seguida, foi feito o peneiramento para a obtenção do material de interesse, sendo ele o material passante na peneira de $19,00 \mathrm{~mm}$ e retido na peneira de $4,76 \mathrm{~mm}$.

Os principais parâmetros de laboratório utilizados para avaliar a aplicação dos RCV em pavimentos foram compactação e California Bearing Ratio (CBR). Estes ensaios foram realizados para cada variação de substituição de RCV no solo. Ainda, foram efetuados ensaios de caracterização básica dos solos e dos RCV. Para tal, utilizaram-se os ensaios de granulometria (ABNT-NBR 7181/84), massa específica dos grãos (DNER-ME 093/1994a), limite de liquidez e plasticidade (DNER-ME 082/1994b e 122/1994c). Adicionalmente, os materiais foram classificados pelo Unified Soil Classification System (USCS) e American Association of State Highway and Transportation Officials (AASHTO) e, para os RCV, foram realizados ensaios de Abrasão Los Angeles, Treton e Fluorescência de Raio X (FRX). A Figura 1 reporta o resíduo na forma de descarte e na forma triturado, passante na peneira de $19,00 \mathrm{~mm}$ e retido na peneira $4,76 \mathrm{~mm}$.

Figura 1: Imagens do RCV coletado nas indústrias de cerâmica (esquerda) e RCV triturado e peneirado (direita)

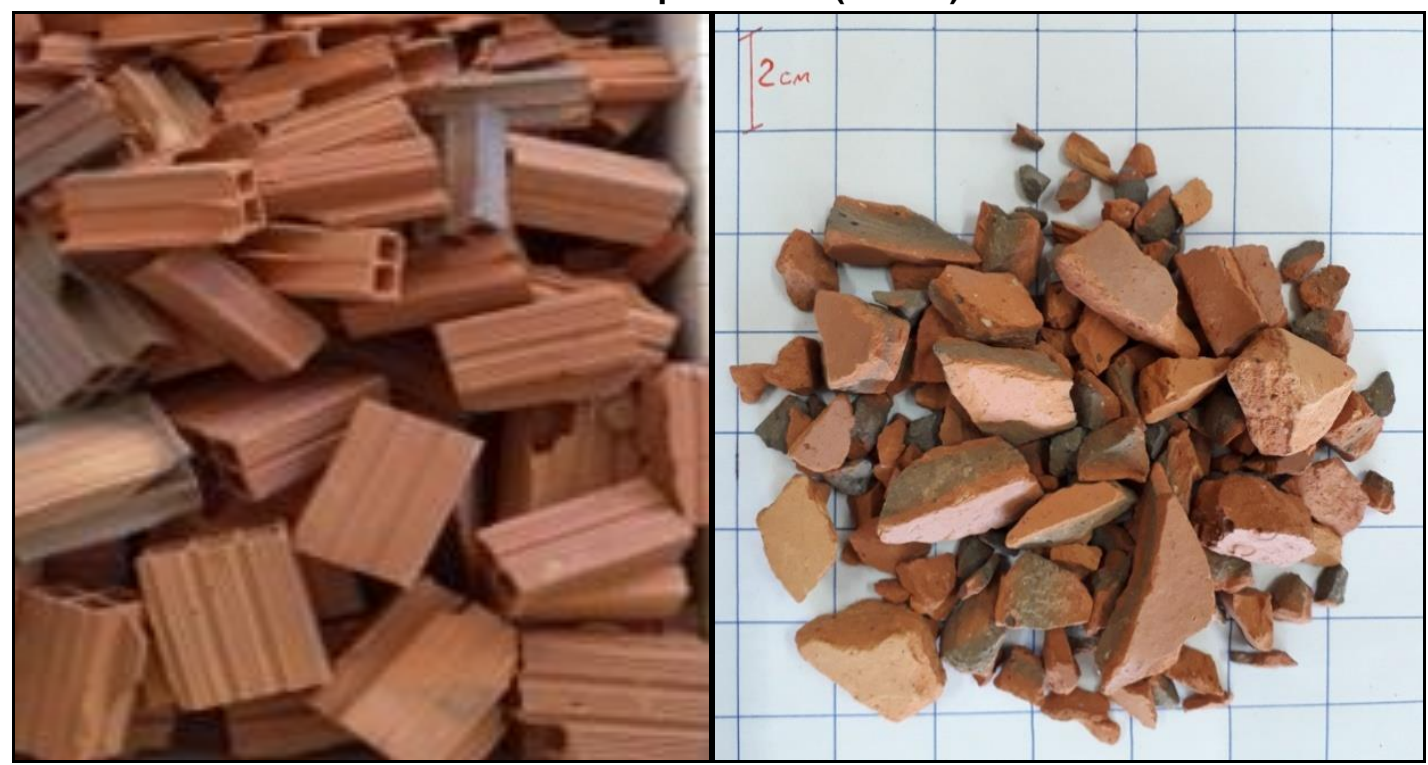

Fonte: Autores, 2019.

Para iniciar a pesquisa, o RCV foi triturado até atingir a granulometria mostrada na Figura 1, conforme descrito anteriormente. Assim, foi investigado o solo puro e o solo com RCV, utilizando taxas de substituição de $30 \%, 50 \%$ e $70 \%$ da massa seca do material, e adicionalmente testou-se o RCV puro, contabilizando cinco espécimes de amostras.

Objetivando o conhecimento da composição mineralógica do RCV, realizou-se o ensaio de Fluorescência de Raios X (FRX) em três amostras.

De modo a compreender as possíveis aplicações dos RCV em camadas de pavimentos, foi necessário realizar ensaios de compactação nas energias normal, intermediária e modificada, seguindo as recomendações do DNER-ME 162/1994d.

Para tanto, procurou-se garantir a homogeneização da mistura entre os materiais até atingir uma uniformidade, principalmente na umidade. Após isso, procederam-se com os ensaios de compactação nas três energias citadas, sendo todas as amostras ensaiadas através de um compactador Proctor/CBR mecânico que, por sua vez, diminui a influência do operador no ensaio, bem como um cilindro CBR de $3229 \mathrm{~cm}^{3}$. 
A partir do ensaio de compactação, foram determinadas as massas específicas secas máximas e as umidades ótimas para cada material. Para estimar a resistência mecânica e compacidade, realizaram-se ensaios de CBR (DNIT-ME 172/2016). Moldaram-se corpos de provas relativos a cada energia de compactação na umidade ótima dos materiais e das misturas. Posteriormente, extensômetros foram acoplados a cada cilindro e, em seguida, imersos em água por 96 horas. No término das 96 horas de imersão, os cilindros foram retirados da água e, após 15 minutos, realizadas as penetrações em uma prensa, contendo um pistão com área de $19,2 \mathrm{~cm}^{2}$ e uma velocidade constante de 0,05 $\mathrm{pol} / \mathrm{min}$. As leituras foram feitas nos tempos determinados pelo DNIT-ME 172/2016) e, em seguida, efetuados os cálculos do CBR.

Sabe-se que a resistência ao desgaste e ao choque está intimamente ligada à ação do tráfego, bem como ao movimento de diversas partículas (DNIT, 2006). Logo, de modo a avaliar as respectivas resistências, o material foi submetido ao ensaio de abrasão Los Angeles e ao ensaio Treton. O primeiro ensaio (DNER-ME 035/98) foi realizado apenas nos RCV, utilizando como carga abrasiva 11 esferas com massa de carga de $4.584 \pm 25$ (g) e uma massa total do material de $5000 \pm 10$ (g). Já o ensaio Treton (DNER-ME 399/99) foi feito também apenas nos RCV, usando 20 grãos do material passante na peneira de $19 \mathrm{~mm}$ e retidos na peneira de $16 \mathrm{~mm}$, para três determinações.

\section{RESULTADOS, ANÁLISE E DISCUSSÃO}

$\mathrm{Na}$ região do Cariri cearense, predomina solos finos; contudo, baseado na classificação AASHTO, estes materiais tendem a ter um bom comportamento como subleito de rodovias. Para Cabalar, Hassan e Abdulnafaa (2016), a adição de materiais granulares aos solos finos tende a melhorar o seu comportamento mecânico, até determinado valor de adição ou substituição. Como neste trabalho investigaram-se cinco espécimes de amostras com percentual de RCV nos solos, busca-se, assim, entender qual configuração de mistura entre solo e RCV tem o melhor comportamento mecânico para as camadas granulares dos pavimentos rodoviários. Desta maneira, a granulometria e a plasticidade podem dar um indicativo da qualidade do material. De acordo com as cinco configurações, a considerar os limites de consistência, as amostras mantiveram 0 comportamento não plástico (NP). Tal fato pode ser justificado em decorrência da alta absorção do material cerâmico e sua alta porosidade, não havendo coesão entre as partículas. Em relação ao solo, esse aspecto se deve à sua característica arenosa e, portanto, não plástica.

Já as distribuições granulométricas do solo puro, RCV puro e solo com RCV, utilizando taxas de substituição de $30 \%, 50 \%$ e $70 \%$, podem ser observadas na Figura 2.

Avaliando as curvas granulométricas, baseadas na classificação USCS, é evidenciado que, com exceção do RCV puro, as demais curvas são mal graduadas. Em função da uniformidade, o RCV puro, o solo puro e solo com $30 \%$ de RCV são muito uniformes, as outras duas amostras são consideradas não uniformes (ver Tabela 1).

Considerando a granulometria, o solo em estudo é classificado na American Association of State Highway and Transportation Officials (AASHTO) como A-2-4, apresentando areia, areia siltosa ou argilosa como materiais predominantes, e na Unified Soil Classification System (USCS), como SP, ou seja, areia mal graduada. Já o RCV foi classificado na AASHTO como A-1-a, contemplando pedra britada, pedregulho e areia como materiais predominantes, e na USCS, como GW, ou seja, pedregulho bem graduado, com coeficiente de uniformidade $(\mathrm{Cu})$ e coeficiente de curvatura (Cc) de 1,52 e 1,41, respectivamente. Considerando a fração fina, a massa específica dos grãos do RCV é de $2,62 \mathrm{~g} / \mathrm{cm}^{3}$, ao passo que o solo confere um valor referente a $2,60 \mathrm{~g} / \mathrm{cm}^{3}$.

Consoante Silva et al. (2017), o fato de a massa especifica do resíduo ser superior à massa do solo natural se deve à sua composição mineralógica, uma vez que se podem denotar óxido de ferro e sílica, os quais conferem ao material uma maior massa específica, como pode ser observado na Tabela 2, a qual apresenta a composição mineralógica do RCV por meio de Fluorescência de Raios X (FRX) realizado em três amostras do material. De acordo com o resultado obtido pelo FRX, além de confirmar o resultado da massa específica dos sólidos, percebe-se que o material analisado, no que diz respeito à percentagem de óxidos de sílica, ferro e alumínio, é classificado como resíduo Classe E pela NBR 12653 (ABNT, 1992). 
Figura 2: Distribuição granulométrica das amostras trabalhadas

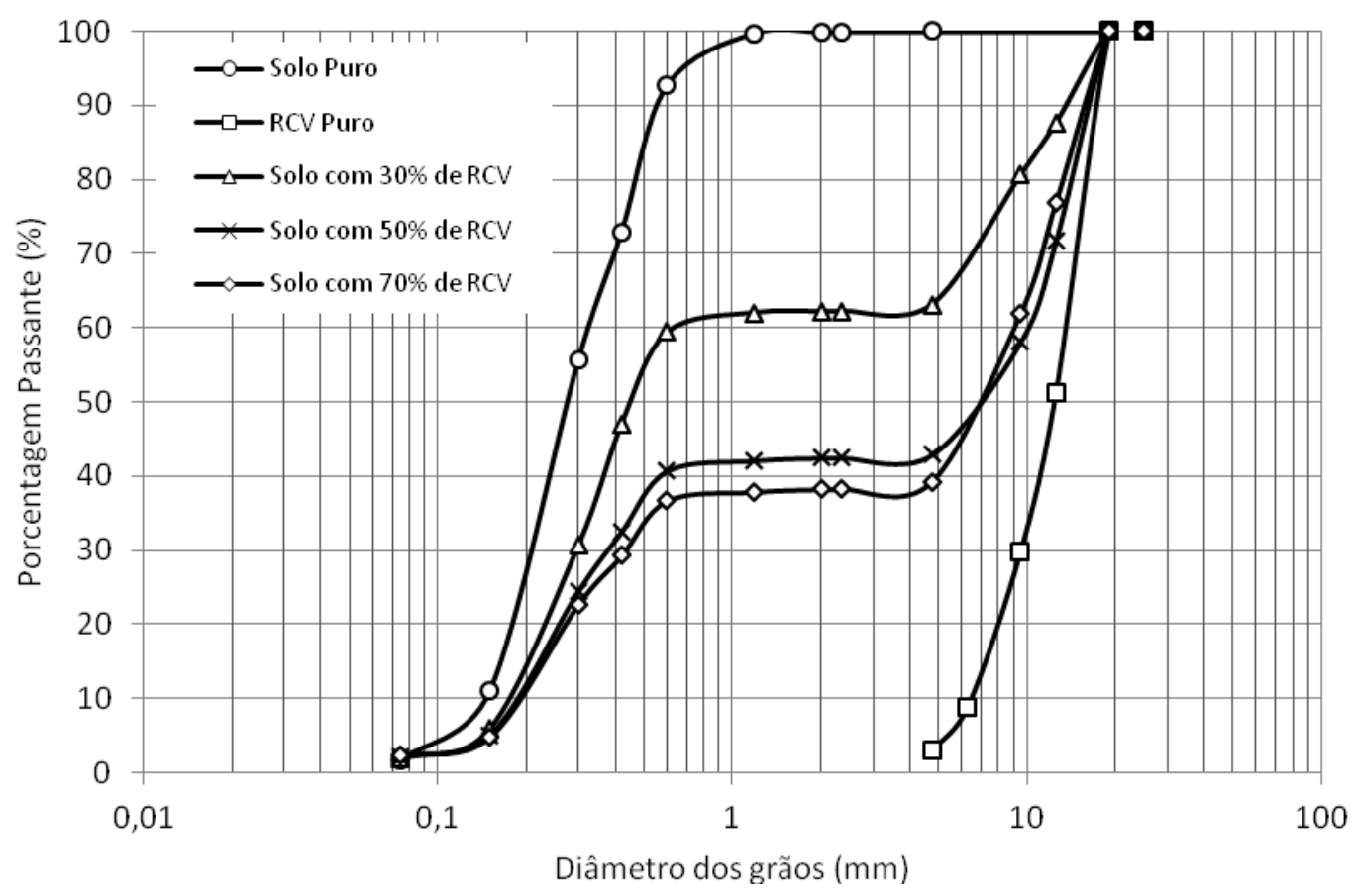

Fonte: Autores, 2019.

Tabela 1: Classificação Cc e Cu das amostras.

\begin{tabular}{c|c|c}
\hline Amostras & $\begin{array}{c}\text { Classificação } \\
\text { (Coeficiente de Curvatura) }\end{array}$ & $\begin{array}{c}\text { Classificação } \\
\text { (Coeficiente de Uniformidade) }\end{array}$ \\
\hline RCV Puro & Bem graduado & Muito uniforme \\
\hline Solo Puro & Mal graduado & Muito uniforme \\
\hline Solo com $30 \%$ de RCV & Mal graduado & Muito uniforme \\
\hline Solo com $50 \%$ de RCV & Mal graduado & Não uniforme \\
\hline Solo com $70 \%$ de RCV & Mal graduado & Não uniforme \\
\hline Fonte: Autores 2019 &
\end{tabular}

Tabela 2 : Composição do resíduo cerâmico em óxidos (FRX).

\begin{tabular}{c|c}
\hline Fórmula & Concentração (\%) \\
\hline $\mathrm{SiO}_{2}$ & 40,32 \\
\hline $\mathrm{Al}_{2} \mathrm{O}_{3}$ & 9,17 \\
\hline $\mathrm{Fe}_{2} \mathrm{O}_{3}$ & 11,18 \\
\hline $\mathrm{K}_{2} \mathrm{O}$ & 4,52 \\
\hline $\mathrm{TiO}_{2}$ & 1,36 \\
\hline $\mathrm{CaO}$ & 1,18 \\
\hline $\mathrm{BaO}$ & 0,11 \\
\hline $\mathrm{Cl}$ & 0,17 \\
\hline $\mathrm{P}_{2} \mathrm{O}_{5}$ & 0,42
\end{tabular}

Fonte: Autores, 2019. 
Considerando a abrasão Los Angeles, realizada apenas no resíduo, encontraram-se valores em torno de $41 \%$ em todas as amostras avaliadas. Convém ressaltar que a NBR 15115 (ABNT, 2004), que preconiza a utilização de agregados reciclados de resíduos sólidos voltados à execução de camadas de pavimentação, não estabelece faixas de valores específicos para a abrasividade de materiais reciclados; porém, de acordo com a NBR 11804 (ABNT, 1991), que trata de materiais para subbase ou base de pavimentos estabilizados, indica um valor de abrasão não excedente a $55 \%$, para sub-bases e bases granulometricamente estabilizadas, e valor referente à brita graduada não excedente a $40 \%$. Segundo o DNIT (2006), a fração graúda das misturas, ou seja, o resíduo deve apresentar um desgaste Los Angeles igual ou inferior a $50 \%$. Ainda, menciona que é possível utilizar materiais com valores superiores a $50 \%$, desde que haja experiências no uso do material.

$\mathrm{Em}$ estudos realizados com outros agregados reciclados, Lima (2008) encontrou uma abrasividade correspondente a $49 \%$ no tocante ao resíduo de construção e demolição (RCD) oriundo de Fortaleza (CE). Carneiro, Brum e Cassa (2001) encontraram um valor equivalente a $45 \%$ em estudos feitos em Salvador (BA). Já Fernandes (2004) estudou o RCD proveniente do Rio de Janeiro (RJ), bem como de Belo Horizonte (MG), encontrando resultados de abrasão Los Angeles de $66 \%$ e $55 \%$, respectivamente.

Por conseguinte, o valor correspondente à abrasividade do RCV em estudo (41\%) corrobora com os valores estabelecidos, atendendo as exigências para seu emprego em camadas de pavimentos.

O ensaio Treton, realizado apenas no RCV puro, visa mensurar a perda de massa do agregado em decorrência do choque ou impacto inerente ao tráfego. A perda de massa por Treton do RCV ficou entre $32,75 \%$ e $67,50 \%$. As especificações e a literatura não estabelecem ou classificam valores ideais para os resultados obtidos pelo Treton, pois tal ensaio é melhor aplicado na comparação e ranqueamento entre materiais. Neste sentido, Ribeiro (2006), Alves, Barroso e Motta (2015) e Carlete, Silva e Castro (2016) encontraram valores de perda de massa por Treton de materiais pétreos britados entre $7,5 \%$ e $42 \%$. Em se tratando de um resíduo mais leve, quando comparado aos agregados pétreos, os valores obtidos pelo Treton são considerados bons, ao passo que superam os valores de algumas pedras britadas citadas na literatura.

Com o intuito de compreender as possíveis aplicações dos RCV em camadas de pavimentos, foi necessário realizar ensaios de compactação nas energias normal, intermediária e modificada, seguindo as recomendações do DNER-ME 162/1994d. As massas específicas secas máximas e as umidades ótimas para cada amostra de solo puro, solo com $30 \%$ de RCV, solo com $50 \%$ de RCV, solo com $70 \%$ de RCV e RCV puro podem ser visualizadas nas Figuras 3 , 4 e 5 , as quais representam os resultados para as energias normal, intermediária e modificada, nesta ordem.

\section{Figura 3: Curvas de compactação do solo, do RCV e suas} misturas na energia normal

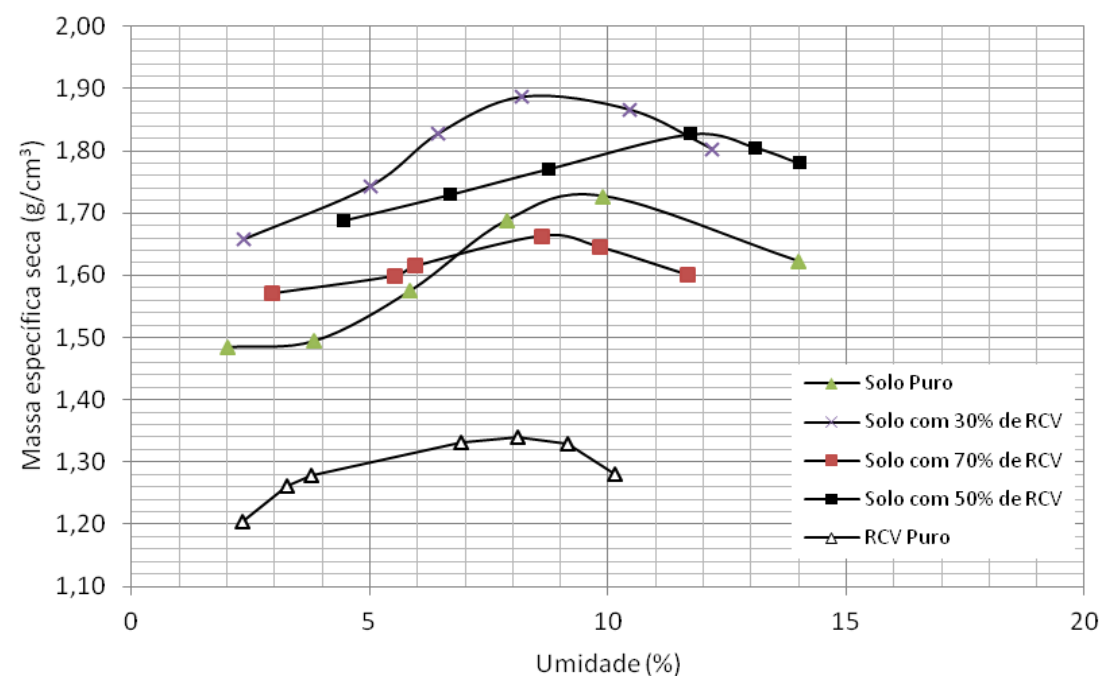

Fonte: Autores, 2019. 
Figura 4 : Curvas de compactação do solo, do resíduo e suas respectivas misturas na energia intermediária

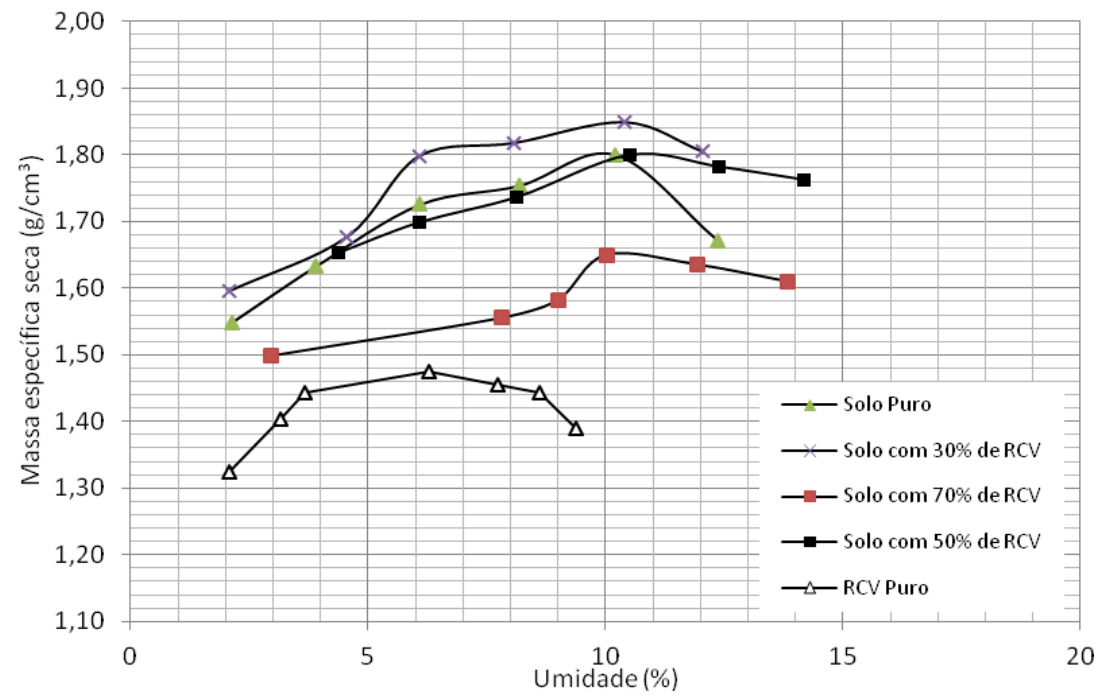

Fonte: Autores, 2019.

Figura 5: Curvas de compactação do solo, do resíduo e suas respectivas misturas na energia modificada

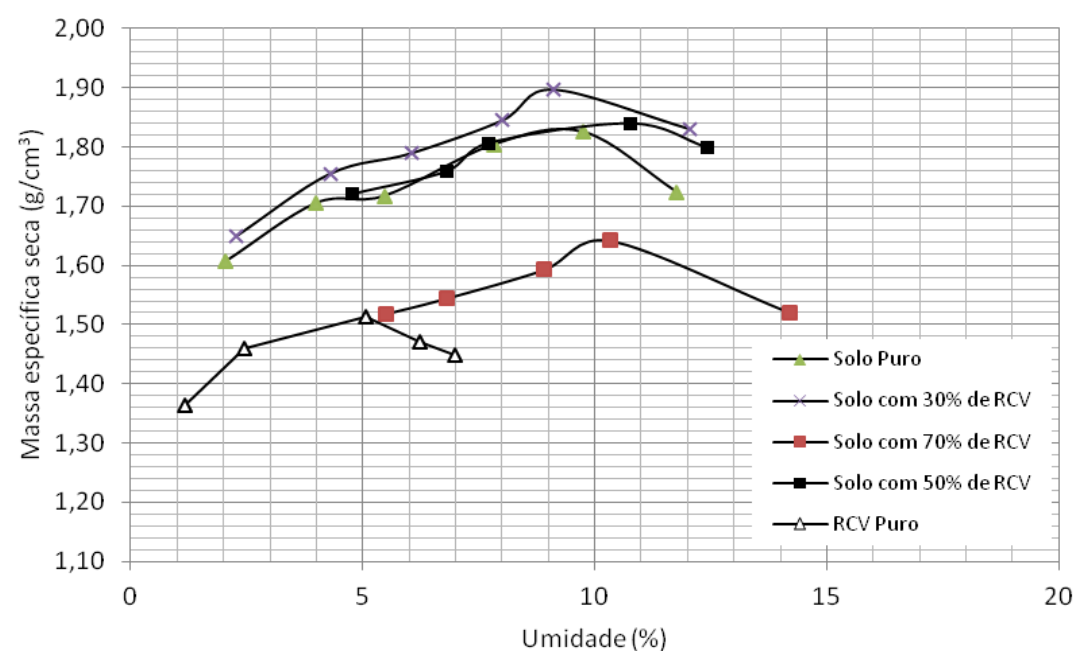

Fonte: Autores, 2019.

De acordo com as curvas obtidas, é possível observar para todas as energias um comportamento similar, no qual o solo com $30 \%$ de RCV tem a maior massa específica seca máxima, e o solo com $50 \%$ de RCV tem a segunda maior massa específica seca máxima, sendo seguido pelo solo puro, solo com $70 \%$ de RCV e, finalmente, pelo RCV puro. Desta forma, com o apoio da Figura 6, pode-se afirmar que a tendência natural é que haja um aumento da massa específica seca máxima em relação ao solo puro, até um limite de $50 \%$ de substituição. A partir desse valor, ocorre um decréscimo na massa específica seca máxima do material. Assim, é esperado que a compacidade e a resistência mecânica do material também sejam incrementadas até esse índice de substituição do solo pelo RCV.

A Figura 7 mostra a variação da umidade ótima do material em relação ao percentual de RCV adicionado ao solo. Nela, observa-se que as maiores umidades ótimas, nas três energias, deram-se na mistura de solo com $50 \%$ de RCV, podendo ainda se verificar que, a partir desse valor, há uma diminuição da umidade ótima com o aumento da quantidade de RCV. 
A partir das massas específicas secas máximas e umidades ótimas para cada amostra de solo puro, solo com $30 \%$ de RCV, solo com $50 \%$ de RCV, solo com $70 \%$ de RCV e RCV puro, fizeram-se os ensaios de CBR. Os resultados podem ser observados na Tabela 3 , para as energias normal, intermediária e modificada.

Figura 6: Variação da massa específica seca máxima com o percentual de RCV para as três energias

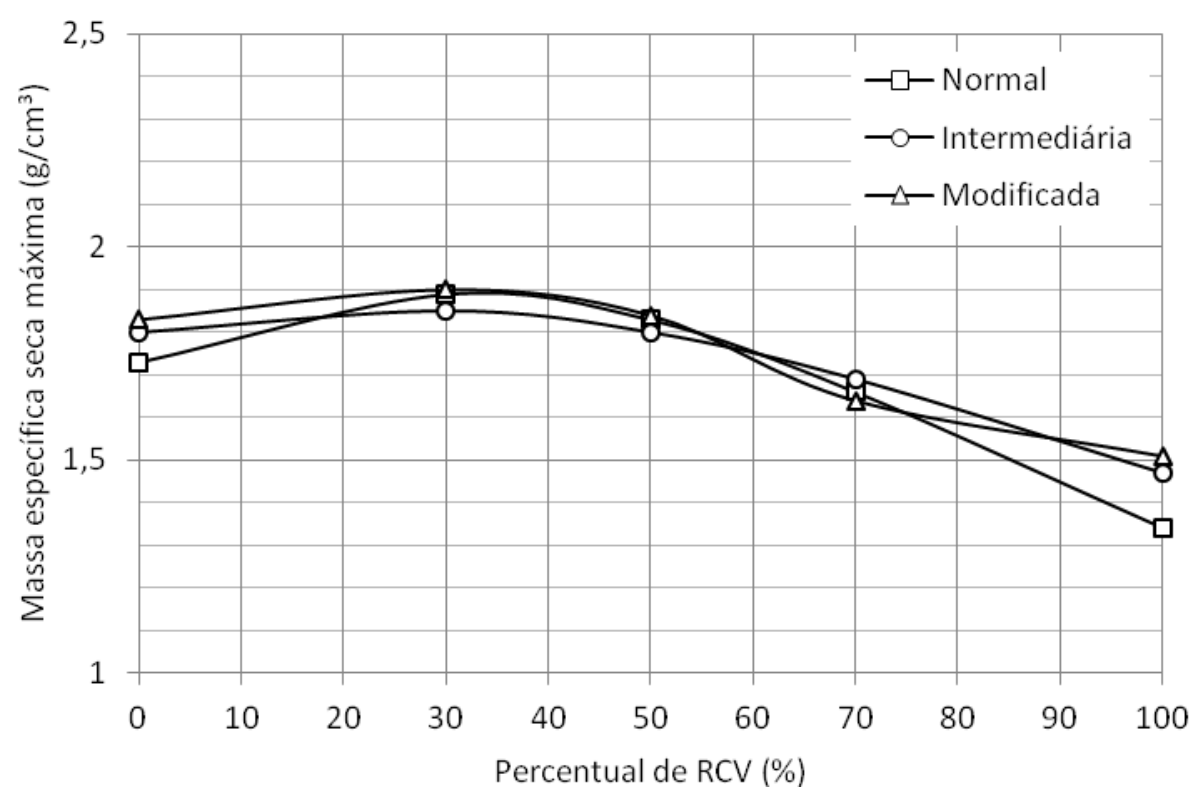

Fonte: Autores, 2019.

Figura 7: Variação da umidade ótima com o percentual de RCV para as três energias

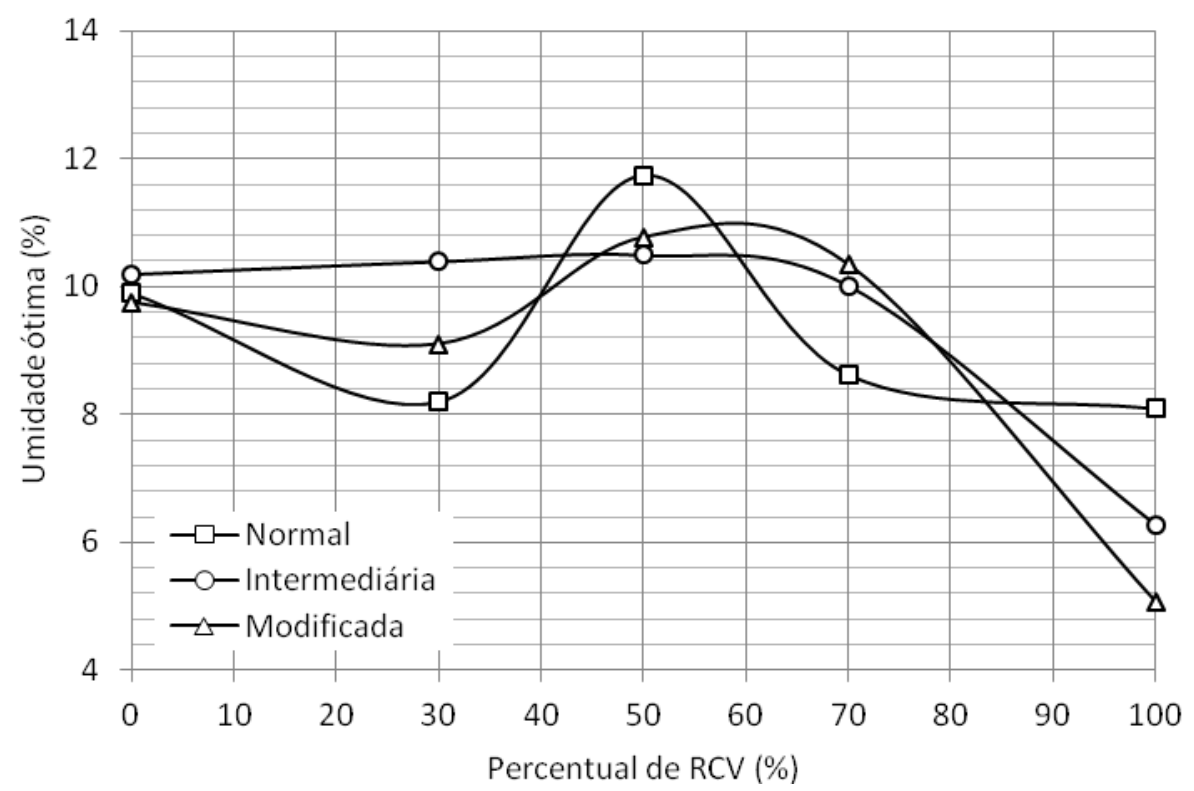

Fonte: Autores, 2019. 
Tabela 3: Valores de CBR das amostras

\begin{tabular}{c|c|c|c|c|c}
\hline Energias & $\begin{array}{l}\text { Solo puro } \\
(\%)\end{array}$ & $\begin{array}{l}\text { Solo com 30\% de } \\
\text { RCV (\%) }\end{array}$ & $\begin{array}{l}\text { Solo com 50\% de } \\
\text { RCV (\%) }\end{array}$ & $\begin{array}{l}\text { Solo com 70\% de } \\
\text { RCV (\%) }\end{array}$ & $\begin{array}{l}\text { RCV puro } \\
(\%)\end{array}$ \\
\hline Normal & 8,44 & 21,37 & 100,31 & 7,84 & 5 \\
\hline Intermediária & 26,31 & 91,87 & 147,3 & 31,58 & 28,65 \\
\hline Modificada & 38 & 40,22 & 218,98 & 200,59 & 34 \\
\hline
\end{tabular}

Fonte: Autores, 2019.

Considerando o referido ensaio, a NBR 15115 (ABNT, 2004) preconiza valores pertinentes ao CBR, bem como a expansão para o emprego de materiais reciclados em projetos rodoviários. Para o reforço de subleito, o CBR deve ser $\geq 12 \%$ e expansão $\leq 1,0 \%$ referentes à energia de compactação normal. Para camadas de sub-base de pavimentos, estes devem ser iguais ou superiores a $20 \%$ e menor ou igual $1,0 \%$ respectivamente, executado na energia intermediária, ao passo que, para camada de base, o CBR deve ser maior ou igual a $60 \%$ e a expansão não pode ser menor do que $0,5 \%$, isso também na energia intermediária.

O DNIT (2006) especifica que materiais empregados em reforço de subleito o valor do CBR deverá ser superior ao do subleito, ou seja, $\geq 2 \%$ com expansão inferior ou igual a $1 \%$. No que diz respeito à sub-base, as especificações são iguais aos da NBR 15115 (ABNT, 2004). Já em relação ao emprego dos materiais em base de pavimentos, o CBR deverá ser $\geq 80 \%$, com expansão $\leq 0,5 \%$, limite de liquidez $\leq 25 \%$ e com índice de plasticidade inferior ou igual a $6 \%$.

De acordo com os resultados obtidos nos ensaios, todas as amostras estudadas conferiram resultados iguais a $0 \%$ no que concerne à expansão. Analisando os resultados de CBR para todas as energias, observa-se que os valores crescem com a substituição de RCV, atingindo, assim, valores máximos para o solo com $50 \%$ de $\mathrm{RCV}$, e a partir disso os valores vão decrescendo. A Figura 8 apresenta o comportamento descrito.

Quanto ao uso dos materiais em pavimentos rodoviários, em relação às especificações e normas vigentes, a pesquisa evidencia que: (i) 0 solo com $30 \%$ de RCV e o solo com $50 \%$ de RCV têm aplicações mais nobres, podendo ser aplicados até em camada de base; (ii) o solo puro e o RCV puro podem ser aplicados em camadas de reforço do subleito e sub-base; e (iii) o solo com $70 \%$ de RCV pode ser aplicado como camadas de reforço do subleito, sub-base e base.
Silva e Carvalho (2017) afirmam que, geralmente, as amostras que apresentam maior massa específica seca máxima os CBR tendem a valores mais elevados. Contudo, foi observado que os maiores CBR resultaram da mistura de solo com $50 \%$ de RCV, e estes apresentaram a segunda maior massa específica seca máxima para as três energias, sendo que a mistura de solo com $30 \%$ de RCV apresentou maior massa específica seca máxima, também para as três energias dentre as espécimes ensaiadas.

O comportamento descrito do material pode ter relação com a quebra do RCV, pois na porcentagem de $30 \%$ de substituição os grãos maiores e mais susceptíveis à quebra ficaram encapsulados no solo, aumentando, assim, a massa específica do material. Já a partir de $50 \%$ de substituição, os grãos ficaram mais expostos aos impactos do soquete, sendo quebrados, aumentando a fração fina e, consequentemente, diminuindo a massa específica da mistura. A quebra do RCV pode ser evidenciada ao observar a Figura 9, a qual ilustra a susceptibilidade do resíduo de cerâmica vermelha à quebra decorrente do processo de compactação.

\section{CONCLUSÃO}

De acordo com os resultados obtidos, em relação à granulometria, ocorreu uma alteração no que diz respeito à distribuição granulométrica à medida que se adiciona o RCV, bem como um aumento considerável do $\mathrm{Cu}$. Considerando tais condições, as misturas de solo com $50 \%$ de RCV e solo com $30 \%$ de RCV se mostraram as melhores, tendo em vista sua adequação a NBR 15115 (ABNT, 2004).

O resíduo apresentou uma boa abrasividade quando comparada com a literatura, bem como com as normas vigentes e o manual de pavimentação. Já em relação à massa específica seca máxima, ocorreu um aumento de acordo com a adição do RCV até o limite de $50 \%$. 
Considerando a capacidade suporte do material, a mistura de solo com $50 \%$ de RCV se mostrou melhor, uma vez que apresentou resultados favoráveis quando compactado em qualquer energia. Já o solo, o resíduo e as demais misturas também se enquadraram no intervalo exigido nas normas dependendo da energia de compactação aplicada. É válido ressaltar que não houve expansibilidade em nenhuma das amostras estudadas.
Em suma, evidencia-se que o resíduo, bem como o solo e suas respectivas misturas podem ser utilizados nas camadas granulares de pavimentos rodoviários, a depender da energia de compactação aplicada, uma vez que os valores de CBR, conforme esperado, variaram com as energias. Assim, a depender da energia aplicada, é possível usar os RCV nas camadas de reforço do subleito, sub-base e base de pavimentos rodoviários.

Figura 8: Variação do CBR com o percentual de RCV para as três energias

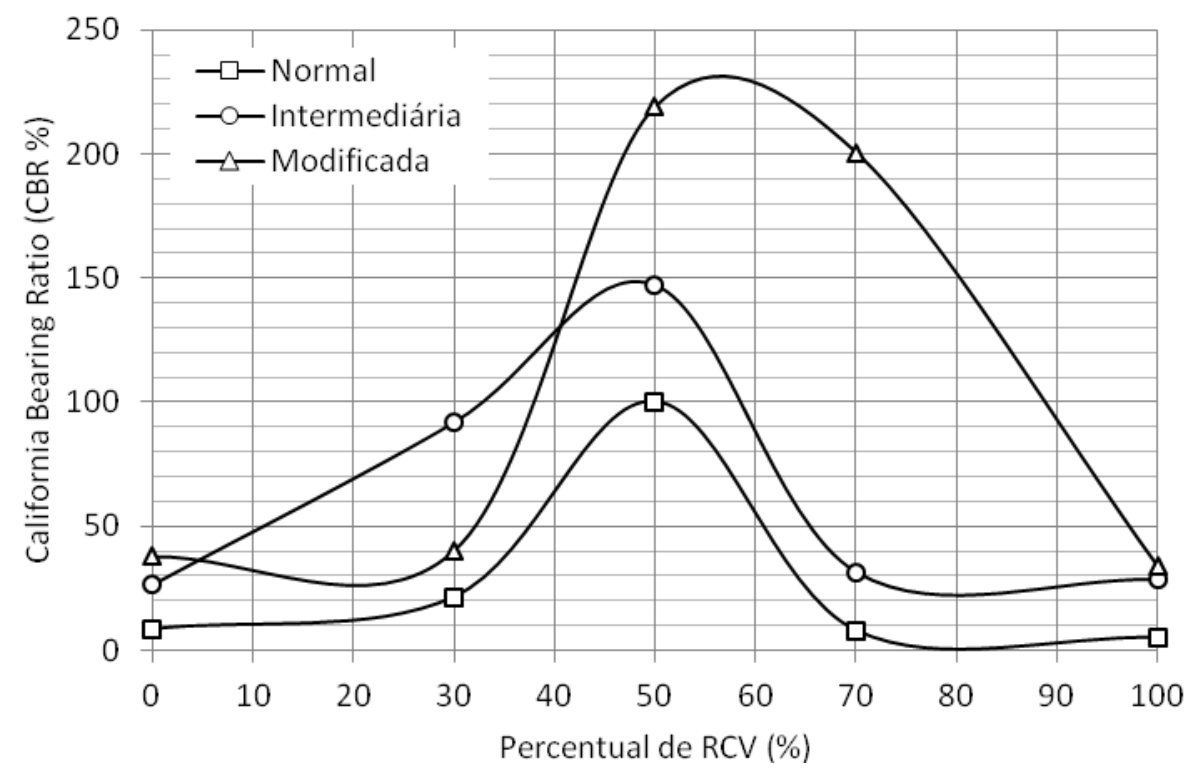

Fonte: Autores, 2019.

Figura 9: Granulometria do RCV pós-compactação nas três energias

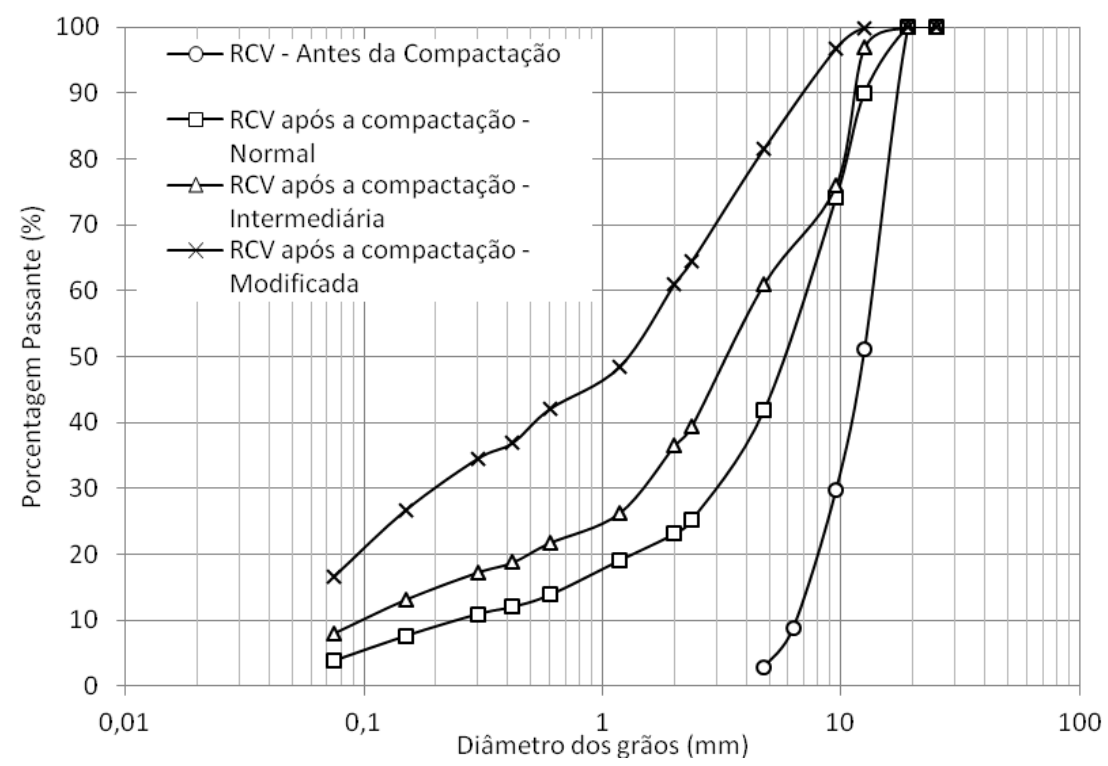

Fonte: Autores, 2019. 


\section{AGRADECIMENTOS}

Os pesquisadores agradecem ao IFCE e ao CNPq, pelo auxílio financeiro e pelas bolsas de iniciação científica concedidas.

\section{REFERÊNCIAS}

ALVES, A. V; VIEIRA, T. F.; DE BRITO, J.; CORREIA, J.R. Mechanical properties of structural concrete with fine recycled ceramic aggregates. Construction and Building Materials, v. 64, p. 103-113, 2014.

ALVES, V.; BARROSO, E. V.; MOTTA, L. M. G. Avaliação da resistência mecânica de agregados da região metropolitana do Rio de Janeiro. In: CONGRESSO BRASILEIRO DE GEOLOGIA DE ENGENHARIA E AMBIENTAL, 15., 2015, Bento Gonçalves. Anais... Bento Gonçalves-RS: ABGE, 2015.

ASSOCIAÇÃO BRASILEIRA DE NORMAS TÉCNICAS. NBR 15115/2004: Agregados reciclados de resíduos sólidos na construção civil - Execução de camadas de pavimentação - Procedimentos. Rio de Janeiro, $2004.10 \mathrm{p}$.

_. NBR 7181/84: Solos - análise granulométrica. Rio de Janeiro, 1984.13 p.

. NBR 12653/92: Materiais pozolânicos. Rio de Janeiro, 1992. 4 p.

NBR 11804/91: Materiais para sub-base ou base de pavimentos estabilizados granulometricamente. Rio de Janeiro, 1991. 3 p.

BANCO DO NORDESTE DO BRASIL. Informe setorial de cerâmica vermelha, $2010.22 \mathrm{p}$.

BETAT, E. F.; PEREIRA, F. M.; VERNEY, J. C. K. Concretos produzidos com resíduos do beneficiamento de ágata: avaliação da resistência à compressão e do consumo de cimento. Matéria, Rio de Janeiro, v. 14, n. 3, p. 1047-1060, 2009.

BINICl, H. Effect of crushed ceramic and basaltic pumice as fine aggregates on concrete mortars properties. Construction and Building Materials, v. 21, p. 1191-1197, 2007.

CABALAR, A. F.; HASSAN, D. I.; ABDULNAFAA, M. D. Use of waste ceramic tiles for road pavement subgrade. Road Materials and Pavement Design, 2016, 16 p.

CARNEIRO, A. P.; BRUM, I. A. S; CASSA, J. C. S. Reciclagem de entulho para produção de materiais de construção. Salvador: EDUFBA; Caixa Econômica
Federal. 312 p, 2001.

CARLETE, B. A.; SILVA, H. V.; CASTRO, N. F. Caracterização de agregados minerais provenientes de resíduos grosseiros de rochas ornamentais. In: SIMPÓSIO DE ROCHAS ORNAMENTAIS DO NORDESTE, 9., 2016. João Pessoa. Anais... João Pessoa-PB, CETEM/MCTI, UFCG, UFPE, Governo do Estado da Paraíba e SEBRAE/PB, 2016. p. 584-592.

DE BRITO, J.; PEREIRA, A. S.; Correia, J. R. Mechanical behaviour of non-structural concrete made with recycled ceramic aggregates. Cement and Concrete Composites, v. 27, p. 429-433, 2005.

DNIT - DEPARTAMENTO NACIONAL DE INFRAESTRUTURA DE TRANSPORTES. 2006. Manual de pavimentação. 3. ed. Diretoria de Planejamento e Pesquisa. Coordenação Geral de Estudos e Pesquisa. Instituto de Pesquisas Rodoviárias. Rio de Janeiro, 2006. 247p.

ME 172/16: Solos - Determinação do Índice de Suporte Califórnia utilizando amostras não trabalhadas. Rio de Janeiro, RJ, 2016. 17 p.

DNER - DEPARTAMENTO NACIONAL DE ESTRADAS DE RODAGEM. ME 093/94. Solos determinação da densidade real. Rio de Janeiro, 1994. 4 p. $1994 a$

ME 082/94: Solos - determinação dos limites de plasticidade. Rio de Janeiro, 1994. 3 p. 1994b

ME 122/94: Solos - determinação do limite de liquidez. Rio de Janeiro, 1994. 7 p. 1994c

ME 162/94: Solos - ensaio de compactação utilizando amostras trabalhadas. Rio de Janeiro, 1994. 7 p. $1994 d$

ME 035/98: Agregados - Determinação da abrasão "Los Angeles". Rio de Janeiro, 1998. 6 p.

ME 399/99: Agregados - determinação da perda ao choque no aparelho Treton. Rio de Janeiro, 1999. $5 \mathrm{p}$.

FERNANDES, C. G. Caracterização mecanística de agregados reciclados de resíduos de construção e demolição dos municípios do Rio de Janeiro e de Belo Horizonte para uso em pavimentação. 2004. 109p. Dissertação (Mestrado) - COPPE-UFRJ, Universidade Federal do Rio de Janeiro, Rio de Janeiro, 2004.

GARCÍA. E.; JUNIOR, M. C.; QUARCIONI, V. A.; CHOTOLI, F. F. Avaliação da atividade pozolânica dos resíduos de cerâmica vermelha. Cerâmica, v. 61, p. 
251-258, 2015.

GARCÍA, J. L. Empleo de residuos de concreto en la construccion de carpetas asfalticas. Master Thesis. Universidad Nacional Autonoma de Mexico, 2014.

HERRADOR, R. Use of recycled construction and demolition waste aggregate for road course surfacing. Journal of Transportation Engineering, v. 138, p. 182-190, 2012.

LEITE, F.C.; MOTA, R. S.; VASCONCELOS, K. L.; BERNUCCI, L. Laboratory evaluation of recycled construction and demolition waste for pavements. Construction and Building Materials, v. 25, p. 29722979, 2011.

LIMA, J. H. C. Utilização de resíduo de construção e demolição para pavimentos urbanos da região metropolitana de Fortaleza. 2008. Dissertação (Mestrado em Engenharia de Transportes) Universidade Federal do Ceará, Fortaleza, 2008.

OLIVEIRA, L. A. P.; GOMES, J. P. C, SANTOS, P. M. $S$. The potential pozzolanic activity of glass and redclay ceramic waste as cement mortars components. Construction and Building Materials, v. 31, p. 197203, 2012.

OSSA, A.; GARCÍ, J. L.; BOTERO, E. Use of recycled construction and emolition waste (CDW) aggregates: a sustainable alternative for the pavement construction industry. Journal of Cleaner Production, v. 135, p. 379-386, 2016.

PACHECO-TORGAL, F.; JALALI, S. Reusing ceramic wastes in concrete. Construction and Building Materials, v. 24, p. 832-838, 2010.
PEREIRA, A. M.; SILVA, C. A. R.; QUEIROZ, D. C. A.; MORAES, M. J. B.; MELGES, J. L. P.; TASHIMA, M. M.; AKASAKI, J. L. Estudo das propriedades mecânicas do concreto com adição de cinza de casca de arroz. Revista Matéria, v. 20, n. 1, p. 227-238, 2015.

REDIVO, I. M. Utilização de resíduo de cerâmica vermelha em misturas com solo para construção de camadas de pavimentos com baixo volume de tráfego. 2011. 160f. Dissertação (Mestrado em Engenharia Civil) - Universidade Federal de Santa Catarina, Florianópolis, 2011.

RIBEIRO, E. M. Caracterização de agregados graúdos do Rio de Janeiro para concreto estrutural. 2006. 355 f. Tese (Doutorado em Ciências em Engenharia Civil) - COOPE, Universidade Federal do Rio de Janeiro, Rio de Janeiro, 2006.

SALES, A. T. C.; ALFERES FILHO, R. S. Efeito do pó de resíduo cerâmico como adição ativa para 0 concreto. Ambiente Construído, Porto Alegre, v. 14, n. 1, p. 113-125, 2014.

SILVA, J. P. S.; CARVALHO, S. B. Uso de resíduos de cerâmica vermelha para o melhoramento de camadas de pavimentos de baixo tráfego em Palmas-TO. Revista Eletrônica de Gestão e Tecnologias Ambientais (GESTA), v. 5, n. 1, p. 41-52, 2017.

SOUZA, W. M.; LIMA, M. M. R.; XAVIER, I. W. P.; RIBEIRO, A. J. A. Diagnóstico da geração de resíduos de cerâmica vermelha na Região Metropolitana do Cariri. In: CONGRESSO TÉCNICO CIENTÍFICO DE ENGENHARIA E DA AGRONOMIA - CONTECC, 76., 2018, Maceió. Anais... Maceió-AL: CREA-AL, 2018. 